\title{
The On-Line Encyclopedia of Integer Sequences
}

\author{
N. J. A. Sloane ${ }^{1}$ \\ May 25, 2018 \\ (To appear in Notices of American Mathematical Society)
}

\begin{abstract}
The recent history of The On-Line Encyclopedia of Integer Sequences (or OEIS), describing developments since 2009, and discussing recent sequences involving interesting unsolved problems and in many cases spectacular illustrations. These include: Peaceable Queens, circles in the plane, the earliest cube-free binary sequence, the EKG and Yellowstone permutations, other lexicographically earliest sequences, iteration of number-theoretic functions, home primes and power trains, a memorable prime, a missing prime, Post's tag system, and coordination sequences.
\end{abstract}

\section{Introduction}

The OEIS (or On-Line Encyclopedia of Integer Sequences ${ }^{2}$ ) is a freely accessible database of number sequences, now in its 54th year, and online since 1995. It contains over 300,000 entries, and for each one gives a definition, properties, references, computer programs, tables, etc., as appropriate. It is widely referenced: a web page ${ }^{3}$ lists over 6000 works that cite it, and often say things like "this theorem would not exist without the help of the OEIS". It has been called one of the most useful mathematical sites in the web.

The main use is to serve as a dictionary or fingerprint file for identifying number sequences (and when you find the sequence you are looking for, you will understand why the OEIS is so popular). If your sequence is not recognized, you see a message saying that if the sequence is of general interest, you should submit it for inclusion in the database. The resulting queue of new submissions is a continual source of lovely problems.

I described the OEIS in a short article in the September 2003 issue of these Notices. The most significant changes since then took place in 2009, when a non-profit foundation ${ }^{4}$ was set up to own and maintain the OEIS, and in 2010 when the OEIS was moved off my home page at AT\&T Labs to a commercial host. The format has also changed: since 2010 the OEIS has been a refereed "wiki". Four people played a crucial role in the transition: Harvey P. Dale and Nancy C. Eberhardt helped set up the Foundation, Russell S. Cox wrote the software, and David L. Applegate helped move the OEIS. The OEIS would probably not exist today but for their help.

\footnotetext{
${ }^{1}$ Neil J. A. Sloane is president of the OEIS Foundation and a visiting scientist at Rutgers Univerity. His email address is njasloane@gmail.com.

${ }^{2}$ http://oeis.org.

${ }^{3}$ http://oeis.org/wiki/Works_Citing_OEIS.

${ }^{4}$ The OEIS Foundation, Inc., http://oeisf .org.
} 
All submissions, of new sequences and updates, are now refereed by volunteer editors. One of the rewards of being an editor is that you see a constant flow of new problems, often submitted by non-mathematicians, which frequently contain juicy-looking questions that are begging to be investigated.

This article will describe a selection of recent sequences, mostly connected with unsolved problems.

Sequences in the OEIS are identified by a 6-digit number prefixed by A. A000001 is the number of groups of order $n$, A000002 is Kolakoski's sequence, and so on. When we were approaching a quarter of a million entries, the editors voted to decide which sequence would become A250000. The winner was the Peaceable Queens sequence, described in the next section, and the runner-up was the "circles in the plane" sequence A250001 discussed in $\S 3$. The $n$th term of the sequence under discussion is usually denoted by $a(n)$.

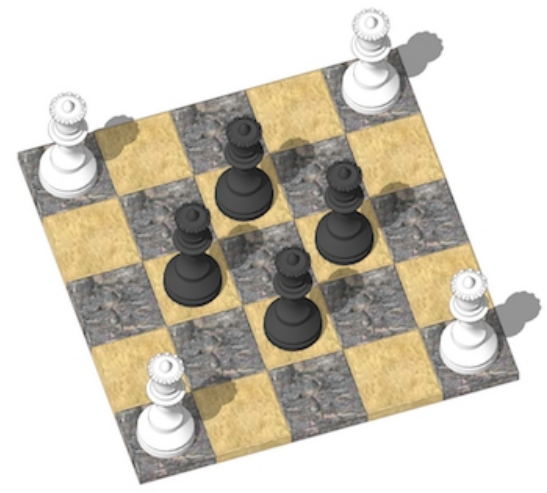

Figure 1: One of three solutions to the Peaceable Queens problem on a $5 \times 5$ board, illustrating $a(5)=4$.

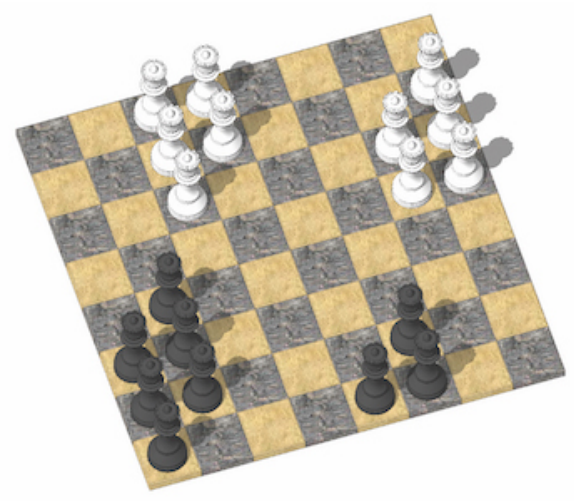

Figure 2: A solution to the Peaceable Queens problem on an $8 \times 8$ board, illustrating $a(8)=9$. (There are actually 10 white queens here but only 9 count since the numbers of white and black queens must be equal. Any one of the white queens could be omitted.) 


\section{Peaceable Queens}

In A250000, $a(n)$ is the maximal number $m$ such that it is possible to place $m$ white queens and $m$ black queens on an $n \times n$ chess board so that no queen attacks a queen of the opposite color. These are peaceable queens. This is a fairly new problem with some striking pictures, an interesting conjecture, and a satisfactorily non-violent theme. It was posed by Robert A. Bosch in 1999, as a variation on the classical problem of finding the number of ways to place $n$ queens on an $n \times n$ board so that they do not attack each other (A000170). It was added to the OEIS in 2014 by Donald E. Knuth, and a number of people have contributed to the entry since then. Only thirteen terms are known:

$\begin{array}{rrrrrrrrrrrrrr}n: & 1 & 2 & 3 & 4 & 5 & 6 & 7 & 8 & 9 & 10 & 11 & 12 & 13 \\ a(n): & 0 & 0 & 1 & 2 & 4 & 5 & 7 & 9 & 12 & 14 & 17 & 21 & 24\end{array}$

Figures $^{5} 1$-4 show examples of solutions for $n=5,8,11$ and (conjecturally) 20.

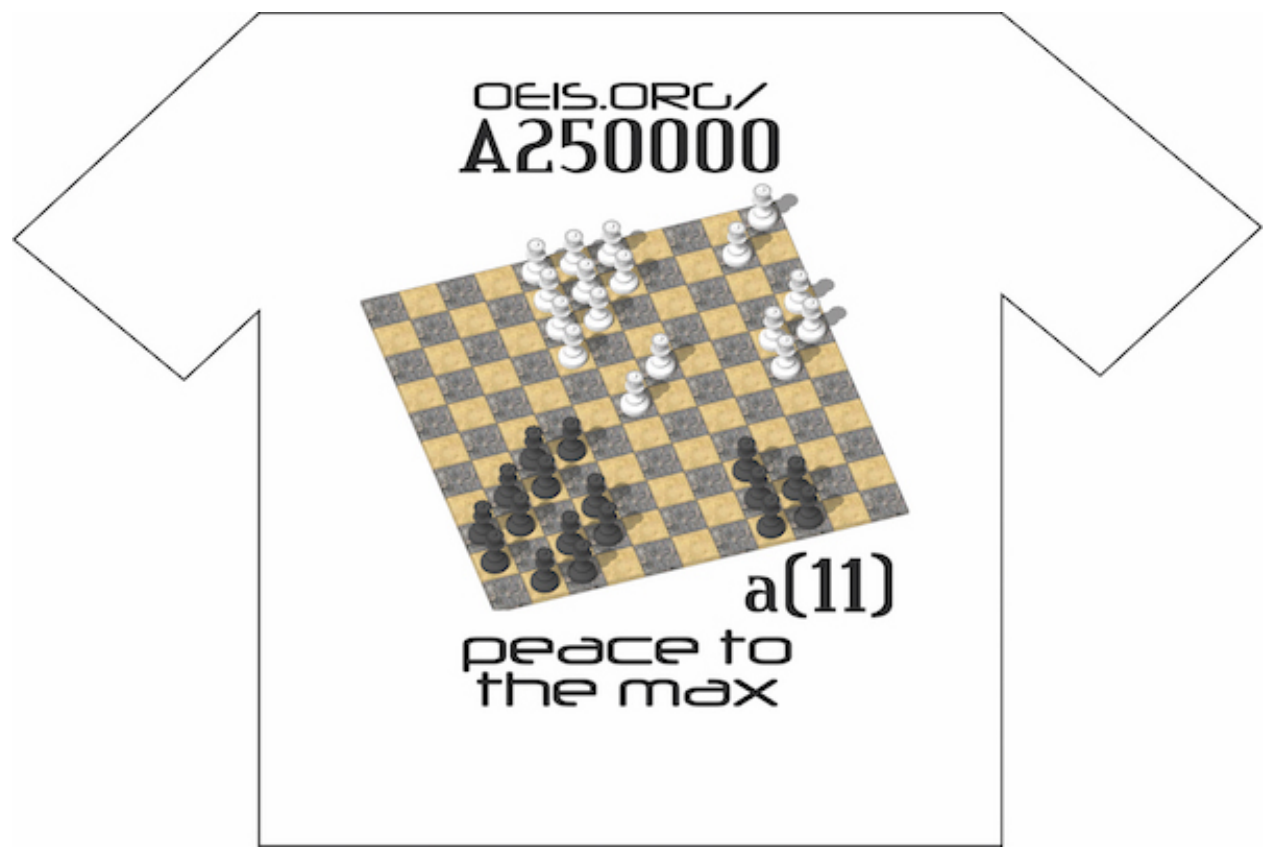

Figure 3: A solution to the Peaceable Queens problem on an $11 \times 11$ board, illustrating $a(11)=17$.

For larger values of $n$, the best solutions presently known were found by Benoît Jubin and concentrate the queens into four pentagonal regions, as shown in Figure 5 (and generalize the arrangement shown in Figure 4). This construction gives a lower bound of $\left\lfloor 7 n^{2} / 48\right\rfloor$, a formula which in fact matches all the best arrangements known so far except $n=5$ and 9 . It would be nice to know if this construction really does solve the problem!

\footnotetext{
${ }^{5}$ The illustrations here are of poor quality because of the limits on file size imposed by the arXiv. For publication-quality illustrations see the version of this article on the author's home page http: //Neilsloane.com.
} 


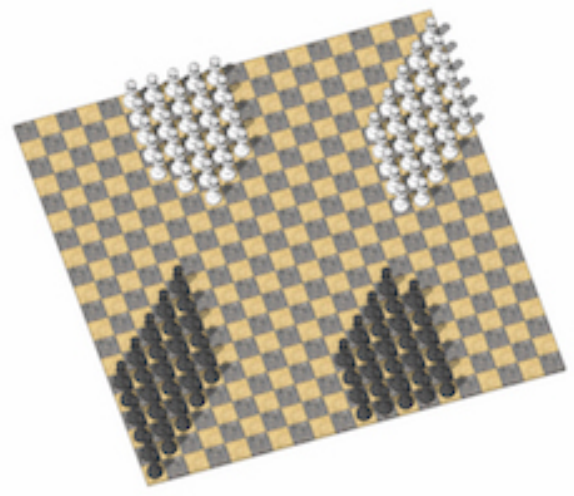

Figure 4: A conjectured solution to the Peaceable Queens problem on a $20 \times 20$ board, found by Bob Selcoe, showing that $a(20) \geq 58$.

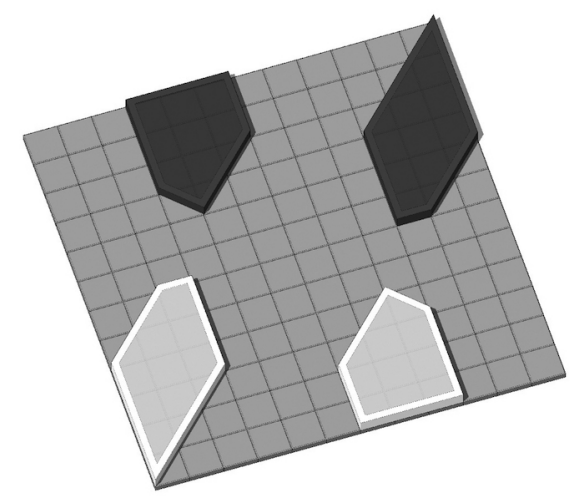

Figure 5: A general construction for the Peaceable Queens problem found by Benoît Jubin, showing that for large $n, a(n) \geq\left\lfloor 7 n^{2} / 48\right\rfloor$, a formula which might be exact for all $n>9$.

\section{Circles in the Plane}

The runner-up in the competition for A250000 is now A250001: here $a(n)$ is the number of ways to draw $n$ circles in the affine plane. Two circles must be disjoint or meet in two distinct points (tangential contacts are not permitted), and three circles may not meet at a point. ${ }^{6}$ The sequence was proposed by Jonathan Wild, a professor of music at McGill University, who found the values $a(1)=1, a(2)=3, a(3)=14, a(4)=173$, and, jointly with Christopher Jones, $a(5)=16951$ (see Figures 6-8).

Wild and Jones have found that there are complications which first appear when five circles are being considered: here there are arrangements which theoretically could exist if one considered only the intersections between circles, but which cannot actually be drawn using circles. For example, start with four circles arranged in a chain, each one overlapping

\footnotetext{
${ }^{6}$ The circles may have different radii. Two arrangements are considered the same if one can be continuously changed to the other while keeping all circles circular (although the radii may be continuously changed), without changing the multiplicity of intersection points, and without a circle passing through an intersection point. Turning the whole configuration over is allowed.
} 


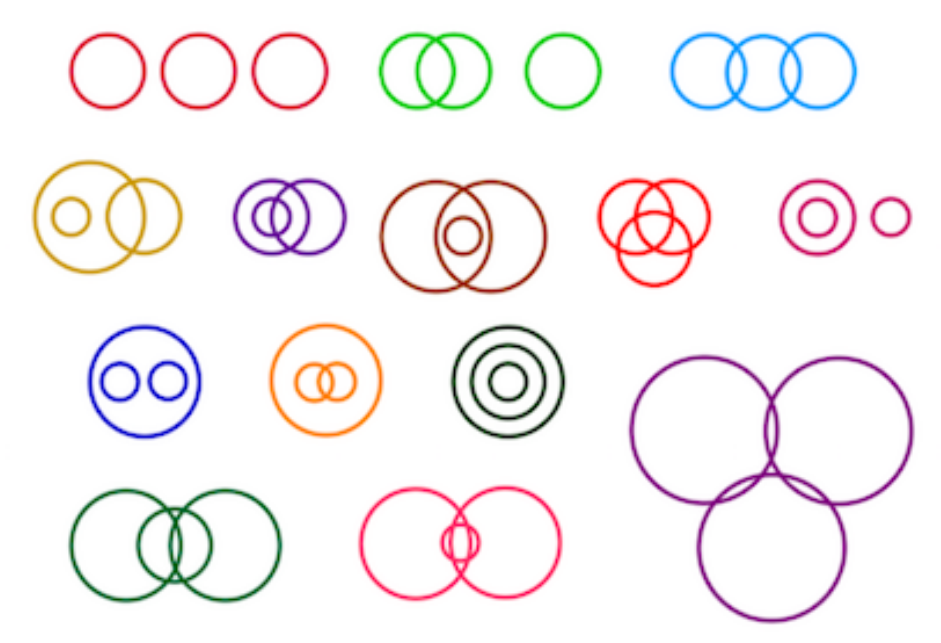

Figure 6: The fourteen ways to draw three circles in the affine plane.
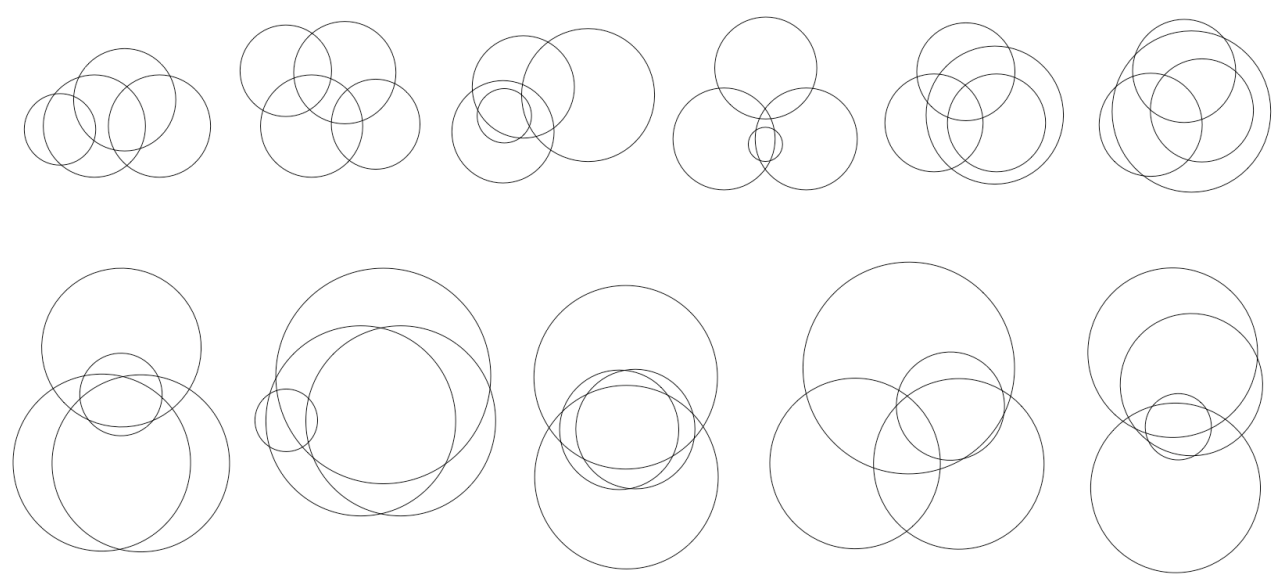

Figure 7: Eight of the 173 ways to draw four circles. For the full set of 173 drawings, see A250001.

its two neighbors, and label the overlaps a, b, c, d (see Figure 9). Suppose we try to add a fifth circle that meets all four circles but avoids their overlaps, encloses overlaps b and d, but does not enclose overlaps a or c. This can be drawn if the fifth circle is flattened to an ellipse, but it can be shown that the arrangement cannot be realized with five circles. There are 26 such unrealizable arrangements of five circles, which can be ruled out by ad hoc arguments.

The delicate configurations like those in Figure 8 are very appealing. It would be interesting to see all 17142 arrangements of five or fewer circles displayed along the Great Wall 


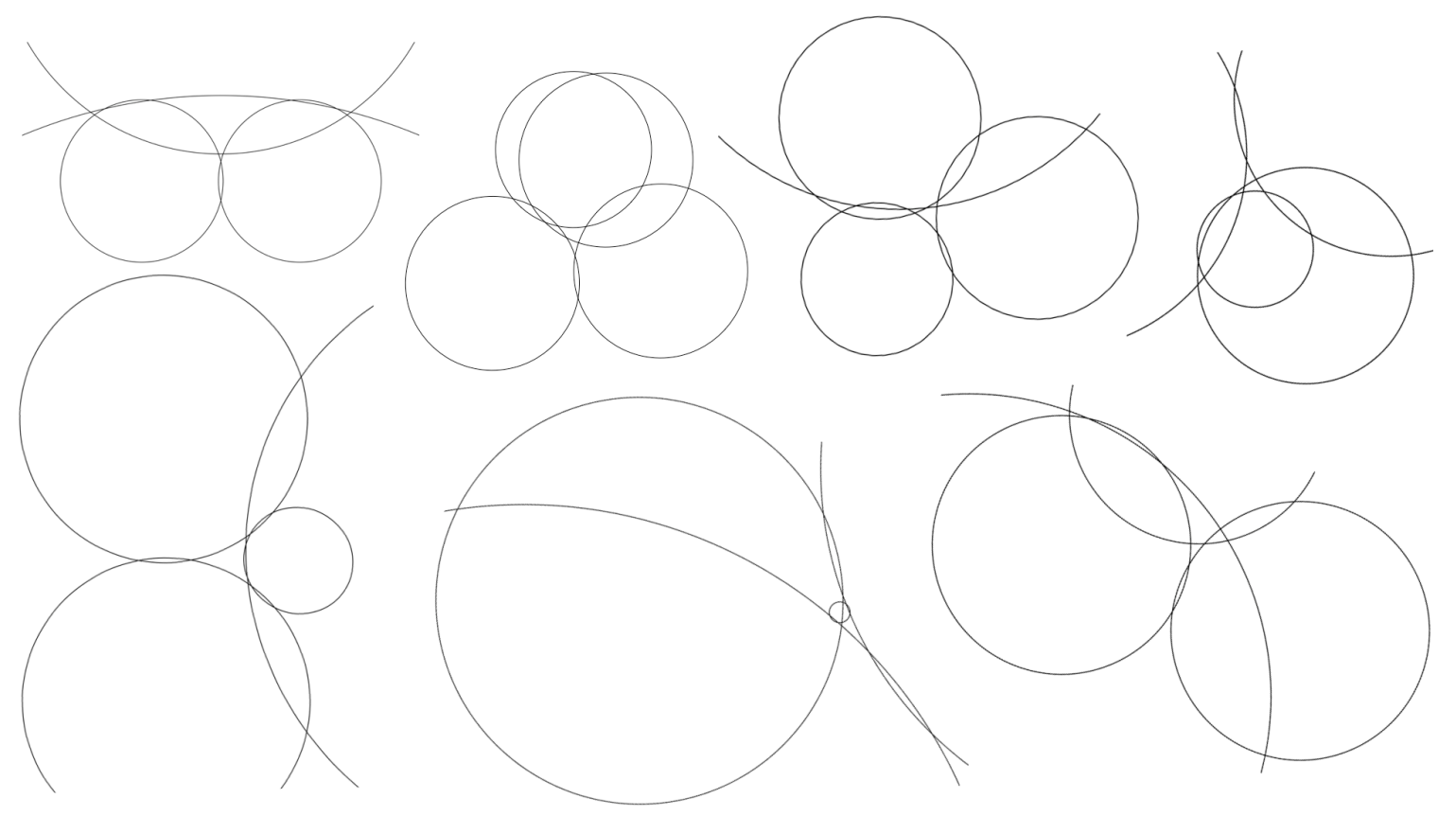

Figure 8: Seven further ways (out of 173) to draw four circles.

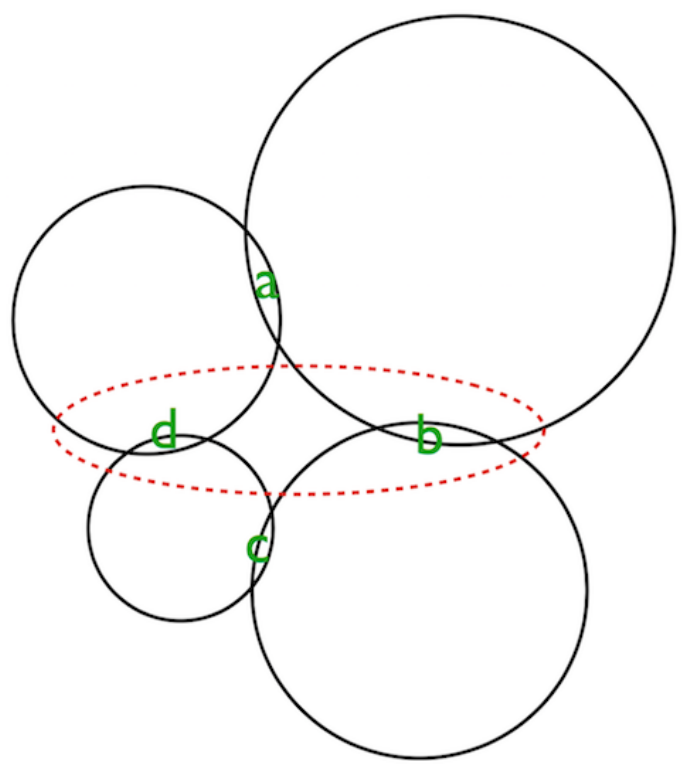

Figure 9: A hypothetical arrangement of five circles that can only be realized if one or more of the circles is distorted.

of China. 


\section{Earliest Cube-Free Binary Sequence}

There is an obvious way to sort integer sequences $a(1), a(2), a(3), a(4), \ldots$ into lexicographic order. A number of recent entries in the OEIS are defined to be the lexicographically earliest sequence of nonnegative or positive integers satisfying certain conditions.

For example, one of the first results in the subject now called "Combinatorics on Words" was Axel Thue's 1912 theorem that the "Thue-Morse sequence"

$$
T=0,1,1,0,1,0,0,1,1,0,0,1,0,1,1,0,1,0,0,1,0,1,1,0,0,1, \ldots
$$

( $\underline{\text { A } 010060}$ ) contains no substring of the form $X X X$, that is, $T$ is cube-free. $T$ can be defined as a fixed point of the mapping $0 \rightarrow 01,1 \rightarrow 10$; alternatively, by taking $a(n)$ to be the parity of the number of $1 \mathrm{~s}$ in the binary expansion of $n .105$ years later, David W. Wilson asked for the lexicographically earliest cube-free sequence of 0 s and 1s. Using a back-tracking algorithm, he found what appear to be the first 10000 terms, which begin

$$
0,0,1,0,0,1,0,1,0,0,1,0,0,1,1,0,0,1,0,0,1,0,1,0,0,1, \ldots
$$

This is now $\mathrm{A} 282317$.

There is no difficulty in showing that the sequence exists. ${ }^{7}$ To see this, make the set $S$ of all infinite binary sequences $a=(a(1), a(2), \ldots)$ into a metric space by defining $d(a, b)$ to be 0 if $a=b$, or $2^{-i}$ if $a$ and $b$ first differ at position $i$. This identifies $S$ with the Cantor set in $[0,1)$. The subset $F \subset S$ of infinite cube-free sequences is nonempty and has an infimum $c$ say. It is easy to show that the complement $S \backslash F$, sequences that contain a cube, is an open set in this topology, so $F$ is closed and $c \in F$.

So far only the first 999 terms of A282317 have been verified to be correct (by showing that there is at least one infinite cube-free sequence with that beginning). The rest of the 10000 terms are only conjectural. It would be nice to know more. In particular, does this sequence have an alternative construction? There is no apparent formula or recurrence, which seems surprising.

\section{The EKG and Yellowstone Sequences}

To continue the "lexicographically earliest" theme, many recent entries in the OEIS are defined to be the lexicographically earliest sequence $a(1), a(2), \ldots$ of distinct positive integers satisfying certain divisibility conditions.

The first task here is usually to show that there are no missing numbers, i.e., that the sequence is a permutation of the positive integers. Sequences of this type were studied in a 1983 paper by Erdős, Freud, and Hegyvári, which included the examples $\underline{\mathrm{A} 036552}(a(2 n)=$ smallest missing number, $a(2 n+1)=2 a(2 n))$ and $\underline{\text { A064736 }}(a(2 n+2)=$ smallest missing number, $a(2 n+1)=a(2 n) \cdot a(2 n+2))$. For these two it is clear that there are no missing numbers. This is less obvious, but still true, for Jonathan Ayres's EKG sequence, A064413, defined to be the lexicographically earliest sequence of distinct positive integers such that

$$
\operatorname{gcd}(a(n-1), a(n))>1 \text { for all } n \geq 3 .
$$

\footnotetext{
${ }^{7}$ Thanks to Jean-Paul Allouche for this argument.
} 


\section{Plot of A064413}

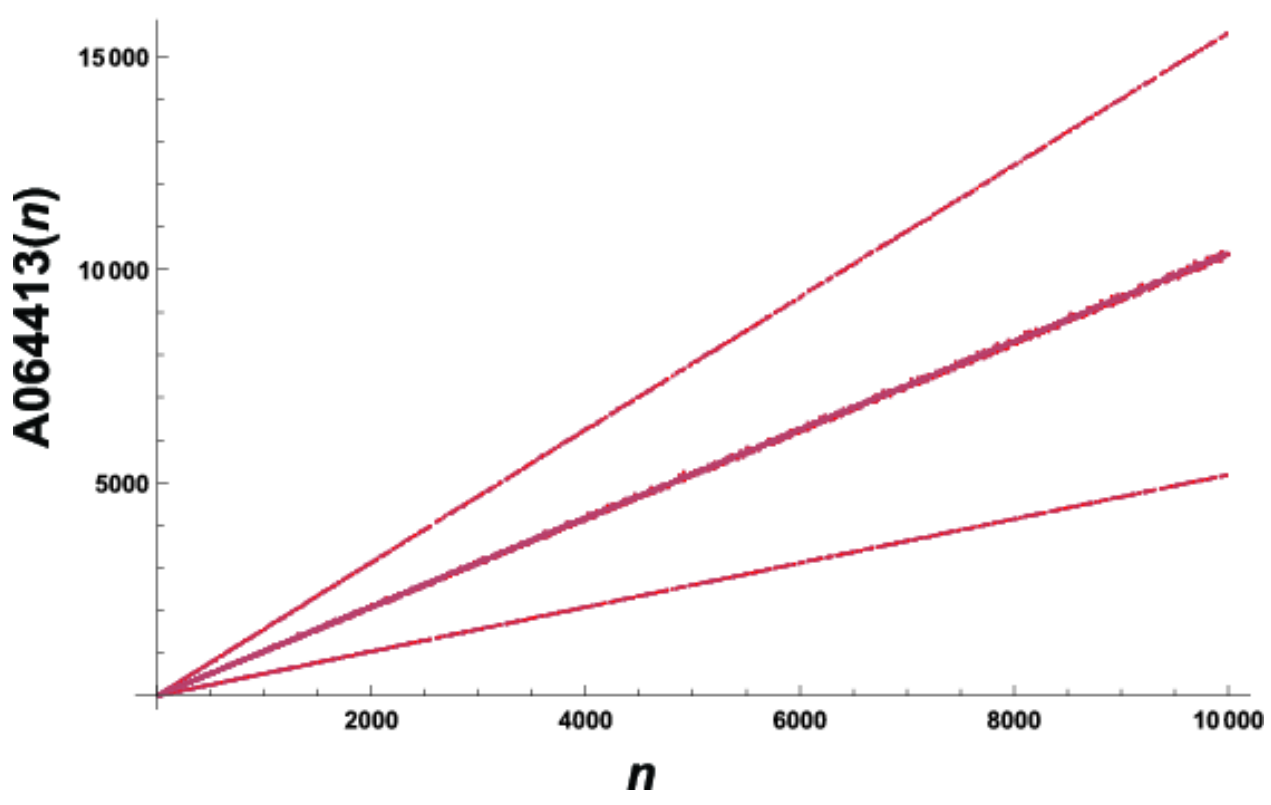

Figure 10: The first 10000 terms of the EKG sequence, so named because locally this graph resembles an EKG. Every number appears exactly once.

This begins

$$
1,2,4,6,3,9,12,8,10,5,15,18,14,7,21,24,16,20,22,11,33,27, \ldots \text {. }
$$

The proof that it is a permutation is omitted-it is similar to the proof for the Yellowstone sequence given below.

Next, one can investigate the rate of growth. In the case of A064413, the points appear to lie roughly on three curved lines (Figure 10), although the following conjecture of Lagarias, Rains, and Sloane (2002) is still open.

Conjecture 1. In the EKG sequence A064413, if a $(n)$ is neither a prime nor three times a prime then

$$
a(n) \sim n\left(1+\frac{1}{3 \log n}\right)
$$

if $a(n)$ is a prime then

$$
a(n) \sim \frac{1}{2} n\left(1+\frac{1}{3 \log n}\right)
$$

and if $a(n)$ is 3 times a prime then

$$
a(n) \sim \frac{3}{2} n\left(1+\frac{1}{3 \log n}\right) .
$$

Furthermore, if the sequence is a permutation, one can also try to study its cycle structure. However, this often leads to very difficult questions, similar to those encountered in studying 
the Collatz conjecture, and we can't do much more than collect experimental data. Typically there is a set of finite cycles, and one or more apparently infinite cycles, but we can't prove that the apparently infinite cycles really are infinite, nor that they are distinct. See the entries for $\underline{\mathrm{A} 064413}$ and $\underline{\mathrm{A} 098550}$ for examples.

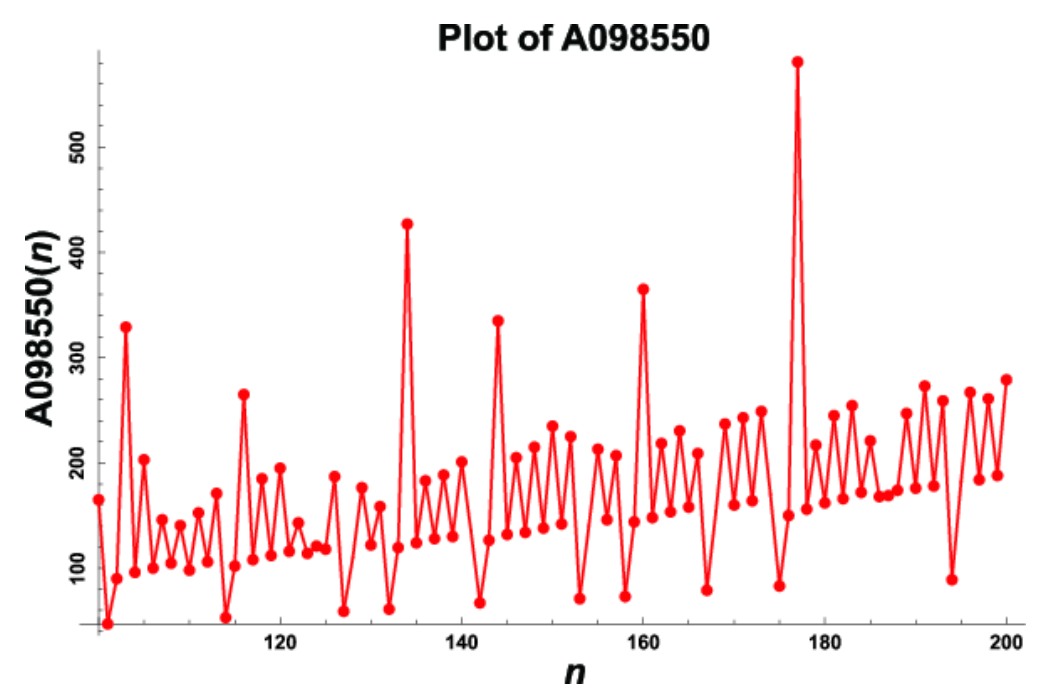

Figure 11: Plot of terms $a(101)$ through $a(200)$ of the Yellowstone sequence. The sequence has a downward spike to $a(n)$ when $a(n)$ is a prime, and larger upward spikes (the "geysers", which suggests the name for this sequence) two steps later.

The definition of the Yellowstone sequence (Reinhard Zumkeller, 2004, A098550, [1]) is similar to that of the EKG sequence, but now the requirement is that, for $n>3$,

$$
\operatorname{gcd}(a(n-2), a(n))>1 \text { and } \operatorname{gcd}(a(n-1), a(n))=1
$$

This begins

$$
1,2,3,4,9,8,15,14,5,6,25,12,35,16,7,10,21,20,27,22,39,11, \ldots
$$

Figure 11 shows terms $a(101)=47$ through $a(200)=279$, with successive points joined by lines.

Theorem 2. The Yellowstone sequence 1098550 is a permutation of the positive integers.

The proof is typical of the arguments used to prove that several similar sequences are permutations, including the EKG sequence above.

Proof. There are several steps.

(i) The sequence is infinite. (For $p a(n-2)$ is always a candidate for $a(n)$, where $p$ is a prime larger than any divisor of $a(i), i<n$.)

(ii) There are infinitely many different primes that divide the terms of the sequence. (If not, there is a prime $p$ such that all terms are products of primes less than $p$. Using (i), find a term $a(n)>p^{2}$, and let $q$ be a common prime factor of $a(n-2)$ and $a(n)$. But now $p q<p^{2}<a(n)$ is a smaller candidate for $a(n)$, a contradiction.) 


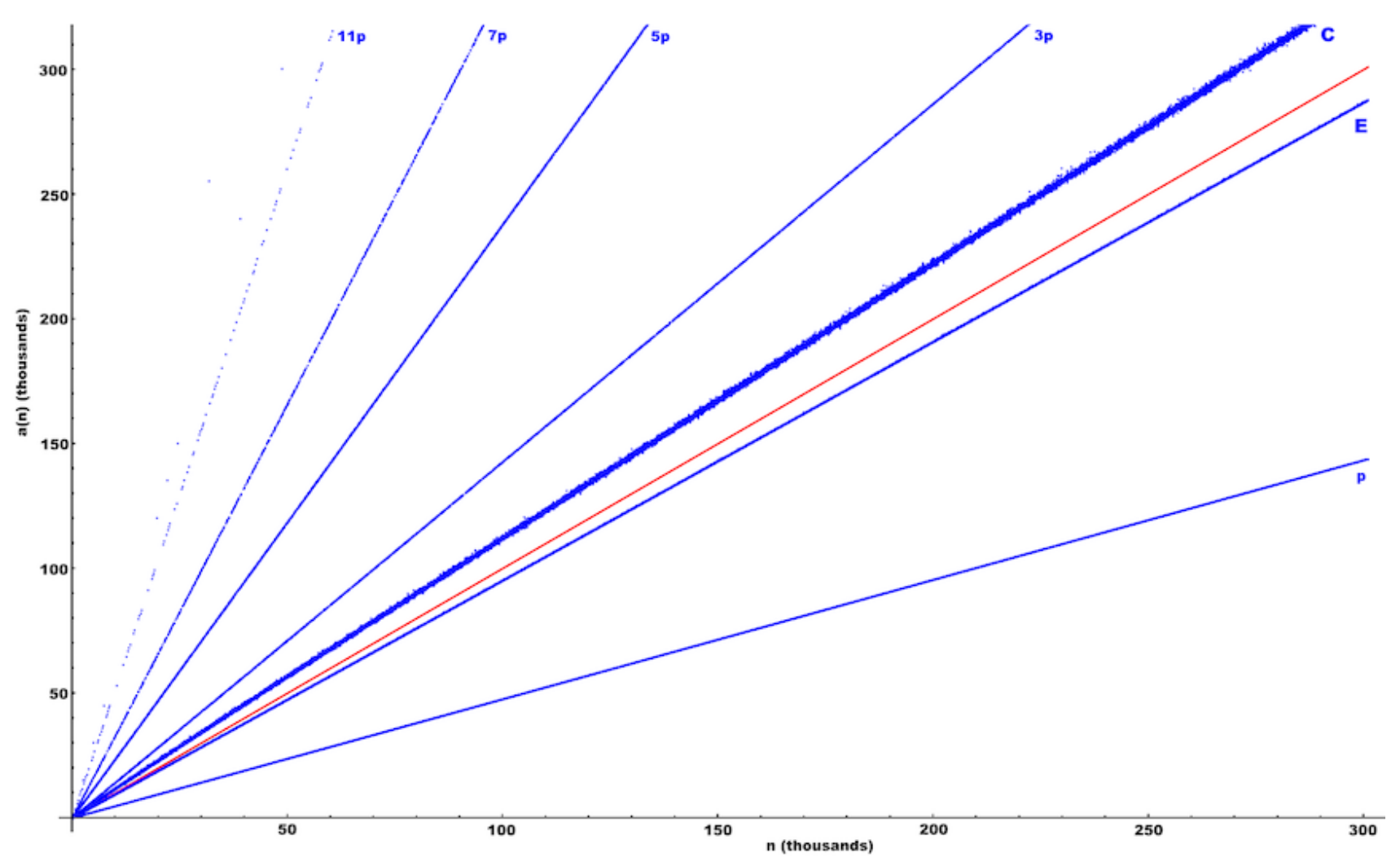

Figure 12: Scatterplot of the first 300,000 terms of the Yellowstone sequence. The primes lie on the lowest line (labeled "p"), the even numbers on the second line ("E"), the majority of the odd composite numbers on the third line ("C"), and the $3 p, 5 p, 7 p, 11 p, \ldots$ points on the higher lines. The lines are not actually straight, except for the red line $f(x)=x$, which is included for reference.

(iii) For any prime $p$, some term is divisible by $p$. (For if not, no prime $q>p$ can divide any $a(n)$ : if $a(n)=k q$ is the first multiple of $q$ to appear, $k p$ would be a smaller candidate for $a(n)$. This contradicts (ii).)

(iv) For any prime $p, p$ divides infinitely many terms. (If not, let $p^{i}$ be larger than any multiple of $p$ in the sequence, and choose a prime $q>p^{i}$. Again we obtain a contradiction.) (v) Every prime $p$ is a term in the sequence. (Suppose not, and using (i), choose $n_{0}$ such that $a(n)>p$ for all $n>n_{0}$. Using (iv), find $a(n)=k p, k>1$, for some $n>n_{0}$. But then $a(n+2)=p$, a contradiction.)

(vi) All numbers appear. For if not, let $k$ be the smallest missing number, and choose $n_{0}$ so that all of $1, \ldots, k-1$ have occurred in $a(1), \ldots, a\left(n_{0}\right)$. Let $p$ be a prime dividing $k$. Since, by (iv), $p$ divides infinitely many terms, there is a number $n_{1}>n_{0}$ such that $\operatorname{gcd}\left(a\left(n_{1}\right), k\right)>1$. This forces

$$
\operatorname{gcd}(a(n), k)>1 \text { for all } n \geq n_{1} .
$$

(If not, there would be some $j \geq n_{1}$ where $\operatorname{gcd}(a(j), k)>1$ and $\operatorname{gcd}(a(j+1), k)=1$, which would lead to $a(j+2)=k$.) But (2) is impossible, because we know from (v) that infinitely many of the $a(n)$ are primes. 
The growth of this sequence is more complicated than that of the EKG sequence. Figure 12 shows the first 300,000 terms, without lines connecting the points. The points appear to fall on or close to a number of distinct curves. There is a conjecture in [1, p. 5] that would explain these curves.

\section{Three Further Lexicographically Earliest Sequences}

Here are three further examples of this type, all of which are surely permutations of the positive integers. For the first there is a proof, for the second there is "almost" a proof, but the third may be beyond reach.

The first (Leroy Quet, 2007, A127202) is the lexicographically earliest sequence of distinct positive integers such that

$$
\operatorname{gcd}(a(n-1), a(n)) \neq \operatorname{gcd}(a(n-2), a(n-1)) \quad \text { for } n \geq 3 .
$$

It begins

$$
1,2,4,3,6,5,10,7,14,8,9,12,11,22,13,26,15,18,16,17,34,19, \ldots
$$

For the second (Rémy Sigrist, 2017, A280864), the definition is

if a prime $p$ divides $a(n)$, then it divides exactly one of $a(n-1)$ and $a(n+1)$, for $n \geq 2$, and the initial terms are

$$
1,2,4,3,6,8,5,10,12,9,7,14,16,11,22,18,15,20,24,21,28,26, \ldots
$$

The proof that the first is a permutation is similar to that for the Yellowstone sequence, although a bit more involved (see A127202). The second struck me as one of those "drop everything and work on this" problems that are common hazards when editing new submissions to the OEIS. However, after several months, I could prove that every prime and every even number appears, and that if $p$ is an odd prime then there are infinitely many odd multiples of $p$ (see A280864 for details), but I could not prove that every odd number appears. The missing step feels like it is only a couple of cups of coffee away, and I'm hoping that some reader of this article will complete the proof.

The third example (Henry Bottomley, 2000, A055265) is the lexicographically earliest sequence of distinct positive integers such that $a(n-1)+a(n)$ is a prime for $n \geq 2$ :

$$
1,2,3,4,7,6,5,8,9,10,13,16,15,14,17,12,11,18,19,22,21,20, \ldots, .
$$

The terms appear to lie on or near the line $a(n)=n$, but the proof that every number appears may be difficult because it involves the gaps between the primes. 


\section{Two-Dimensional Lexicographically Earliest Arrays}

The OEIS is primarily a database of sequences $\left(a_{n}, n \geq n_{0}\right)$. However, triangles of numbers are included by reading them by rows. Pascal's triangle becomes 1, 1, 1, 1, 2, 1, 1,3,3,1, $1,4,6,4,1, \ldots$, which (without the extra spaces) is A007318. Doubly-indexed arrays $\left(T_{m, n}\right.$, $m \geq m_{0}, n \geq n_{0}$ ) are converted to sequences by reading them by antidiagonals (in either the upwards or downwards directions, or both). So an array $\left(T_{m, n}, m \geq 0, n \geq 0\right)$ might become $T_{0,0}, T_{1,0}, T_{0,1}, T_{2,0}, T_{1.1}, T_{0,2}, \ldots$ For example, the table of Nim-sums $m \oplus n$ :

$\begin{array}{lllllllll}0 & 1 & 2 & 3 & 4 & 5 & 6 & 7 & \ldots \\ 1 & 0 & 3 & 2 & 5 & 4 & 7 & 6 & \ldots \\ 2 & 3 & 0 & 1 & 6 & 7 & 4 & 5 & \ldots \\ 3 & 2 & 1 & 0 & 7 & 6 & 5 & 4 & \ldots \\ 4 & 5 & 6 & 7 & 0 & 1 & 2 & 3 & \ldots \\ 5 & 4 & 7 & 6 & 1 & 0 & 3 & 2 & \ldots \\ 6 & 7 & 4 & 5 & 2 & 3 & 0 & 1 & \ldots \\ 7 & 6 & 5 & 4 & 3 & 2 & 1 & 0 & \ldots \\ . & . & . & . & . & . & . & . & \ldots\end{array}$

produces the sequence $\underline{\text { A003987: }}$

$$
0,1,1,2,0,2,3,3,3,3,4,2,0,2,4,5,5,1,1,5,5,6,4,6,0,6,4,6, \ldots .
$$

Doubly-indexed doubly-infinite arrays $\left(T_{m, n}, m \in \mathbb{Z}, n \in \mathbb{Z}\right)$ can become sequences by reading them in a spiral around the origin, in say a counter-clockwise direction: $T_{0,0}, T_{1,0}, T_{1,1}, T_{0,1}$, $T_{-1,1}, T_{-1,0}, T_{-1,-1}, T_{0,-1}, \ldots$ (cf. Figure 13).

There are many "lexicographically earliest" versions of these arrays. For example, the Nim-sum array (3) has an equivalent definition: scan along upwards antidiagonals, filling in each cell with the smallest nonnegative number that is neither in the row to the left of that cell nor in the column above it.

A variation on the Nim-sum array was proposed by Alec Jones in 2016, as a kind of "infinite Sudoku array". This array $\left(T_{m, n}, m \geq 0, n \geq 0\right)$ is to be filled in by upwards antidiagonals, always choosing the smallest positive integer such that no row, column, diagonal, or antidiagonal contains a repeated term. The top left corner of the array is:

$\begin{array}{ccccccccc}1 & 3 & 2 & 6 & 4 & 5 & 10 & 11 & \ldots \\ 2 & 4 & 5 & 1 & 8 & 3 & 6 & 12 & \ldots \\ 3 & 1 & 6 & 2 & 9 & 7 & 5 & 4 & \ldots \\ 4 & 2 & 3 & 5 & 1 & 8 & 9 & 7 & \ldots \\ 5 & 7 & 1 & 4 & 2 & 6 & 3 & 15 & \ldots \\ 6 & 8 & 9 & 7 & 5 & 10 & 4 & 16 & \ldots \\ 7 & 5 & 4 & 3 & 6 & 14 & 8 & 9 & \ldots \\ 8 & 6 & 7 & 9 & 11 & 4 & 13 & 3 & \ldots \\ . & . & . & . & . & . & . & . & \ldots\end{array}$

The resulting sequence $(\underline{\mathrm{A} 269526})$ is

$$
1,2,3,3,4,2,4,1,5,6,5,2,6,1,4,6,7,3,2,8,5,7,8,1,5,9,3,10, \ldots \text {. }
$$


This array has many interesting properties. If we subtract 1 from each entry, the entries are the Nim-values for a game played with two piles of counters, of sizes $m$ and $n$, and reminiscent of Wythoff's game (see A004481, A274528).

But the main question about the array (4) is, are the individual rows, columns, and diagonals of this array permutations of $\mathbb{N}$ ? (The antidiagonals are obviously not, since they are finite sequences.) It is easy to see that each column is a permutation. In column $c \geq 0$, a number $k$ will eventually be the smallest missing number and will appear in some cell in that column, unless there is a copy of $k$ to the North-West, West, or South-West of that cell. But there are at most $c$ copies of $k$ in all the earlier columns, so eventually $k$ will appear.

The rows are also permutations, although the proof is less obvious. Consider row $r \geq 0$, and suppose $k$ never appears. There are at most $r$ copies of $k$ in the earlier rows, and these can affect only a bounded portion of row $r$. Consider a cell $(r, n), n \geq 0$ large. If $k$ is not to appear in that cell, there must be a copy of $k$ in the antidiagonal to the South-West. So in the triangle bounded by row $r$, column 0 , and the antidiagonal through $(r, n)$, there must be at least $n+1-r$ copies of $k$. Imagine these $k$ s replaced by chess queens. By construction they are mutually non-attacking. But it is known ([6, Problem 252], or A274616) that on a triangular half-chessboard of side $n$, there can be at most $2 n / 3+1$ mutually non-attacking queens, which for large $n$ leads to a contradiction.

As to the diagonals, although they appear to be permutations, this is an open question. The argument using non-attacking queens breaks down because the diagonal of the halfchessboard contains only half as many squares as the sides. Even the main diagonal, A274318,

$$
1,4,6,5,2,10,8,3,7,9,16,26,29,22,20,23,28,38,12,32,46,13,14,11,15, \ldots
$$

is not presently known to be a permutation of $\mathbb{N}$.

The spiral version of this array is even more frustrating. This array $((T(m, n), m \in \mathbb{Z}, n \in$ $\mathbb{Z}), \underline{A 274640}$, proposed by Zak Seidov and Kerry Mitchell in June 2016), is constructed in a counterclockwise spiral, filling in each cell with the smallest positive number such that no row, column, or diagonal contains a repeated term (Figures 13, 14). ("Diagonal" now means any line of cells of slope \pm 1 .)

Although it seems very plausible that every row, column, and diagonal is a permutation of $\mathbb{N}$, now there are no proofs at all. The eight spokes through the center are sequences A274924-A274931. For example, the row through the central cell is

$$
\ldots, 14,25,13,17,10,15,7,6,5,3,1,2,4,8,11,12,16,9,19,24,22, \ldots \text {, }
$$

which is $\underline{\mathrm{A} 274928}$ reversed followed by $\underline{\mathrm{A} 274924}$. Is it a permutation of $\mathbb{N}$ ? We do not know.

\section{Fun With Digits}

Functions of the digits of numbers have always fascinated people, ${ }^{8}$ and one such function was in the news in 2017. The idea underlying this story and several related sequences is to

\footnotetext{
${ }^{8}$ Although in A Mathematician's Apology, G. H. Hardy, referring to the fact that 1089 and 2178 are the smallest numbers which when written backwards are nontrivial multiples of themselves (cf. A008919), remarked that this fact was "likely to amuse amateurs", but was not of interest to mathematicians.
} 


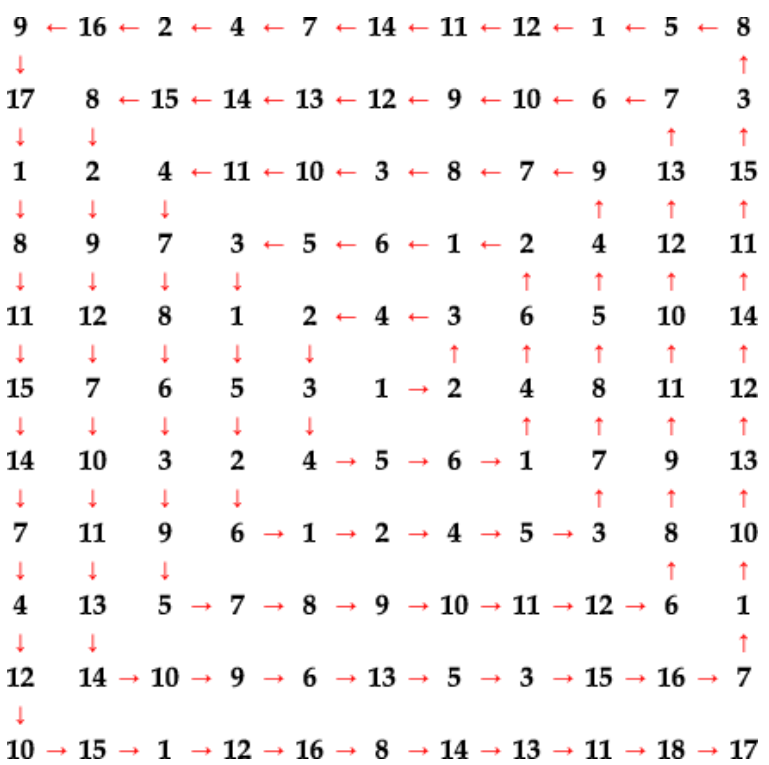

Figure 13: A274640: choose smallest positive number so that no row, column, or diagonal contains a repeat. Are the rows, columns, diagonals permutations of $\mathbb{N}$ ?

start with some simple function $f(n)$ of the digits of $n$ in some base, iterate it, and watch what happens.

For the first example we write $n$ as a product of prime powers, $n=p_{1}^{e_{1}} p_{2}^{e_{2}} \cdots$ with the $p_{i}$ in increasing order, and define $f(n)$ to be the decimal concatenation $p_{1} e_{1} p_{2} e_{2} \ldots$, where we omit any exponents $e_{i}$ that are equal to 1 . So $f(7)=f\left(7^{1}\right)=7, f(8)=f\left(2^{3}\right)=23$.

The initial values of $f(n)(\underline{\mathrm{A} 080670})$ are

$$
\begin{array}{ccccrrrrrrrrrrrrrrrr}
n: & 1 & 2 & 3 & 4 & 5 & 6 & 7 & 8 & 9 & 10 & 11 & 12 & 13 & 14 & 15 & 16 & 17 & 18 & \ldots \\
f(n): & 1 & 2 & 3 & 22 & 5 & 23 & 7 & 23 & 32 & 25 & 11 & 223 & 13 & 27 & 35 & 24 & 17 & 232 & \ldots
\end{array}
$$

If we start with a positive number $n$ and repeatedly apply $f$, in many small cases we rapidly reach a prime (or 1). ${ }^{9}$ For example, $9=3^{2} \rightarrow 32=2^{5} \rightarrow 25=5^{2} \rightarrow 52=2^{2} 13 \rightarrow 2213$, a prime. Define $F(n)$ to be the prime that is eventually reached, or -1 if the iteration never reaches a prime. The value -1 will occur if the iterates are unbounded or if they enter a cycle of composite numbers. The initial values of $F(n)(\underline{\mathrm{A} 195264})$ are

$$
1,2,3,211,5,23,7,23,2213,2213,11,223,13,311,1129,233,17,17137,19, \ldots \text {. }
$$

$F(20)$ is currently unknown (after 110 steps the trajectory of 20 has stalled at a 192-digit number which has not yet been factored). At a DIMACS conference in October 2014 to celebrate the 50th anniversary of the start of what is now the OEIS, John H. Conway offered $\$ 1000$ for a proof or disproof of his conjecture that the iteration of $f$ will always reach a prime.

However, in June 2017 James Davis found a number $D_{0}=13532385396179$ whose prime factorization is $13 \cdot 53^{2} \cdot 3853 \cdot 96179$, and so clearly $f\left(D_{0}\right)=D_{0}$ and $F\left(D_{0}\right)=-1$.

\footnotetext{
${ }^{9}$ In what follows we will tacitly assume $n \geq 2$, to avoid having to repeatedly say "(or 1 )".
} 


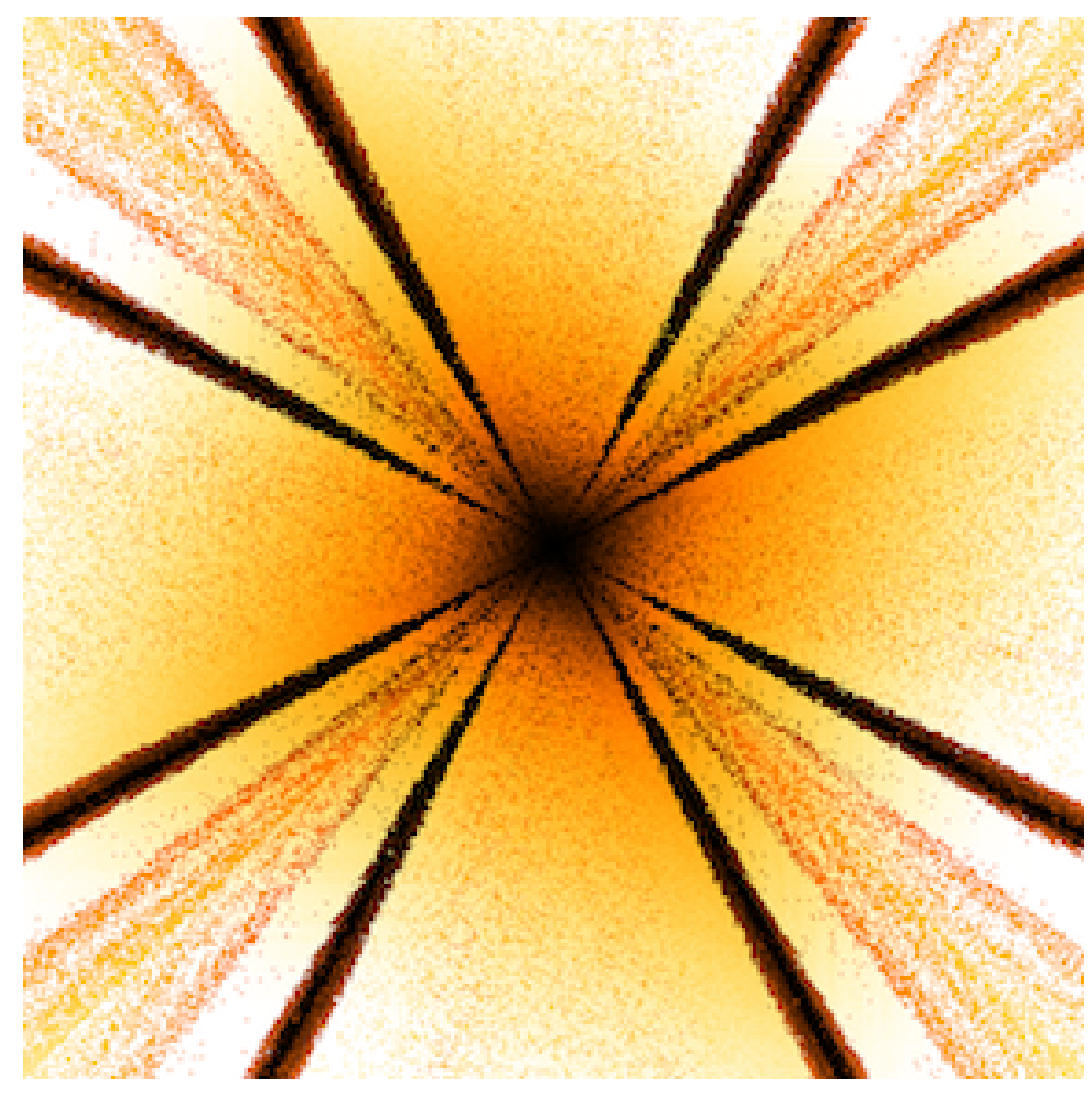

Figure 14: Colored representation of central $200 \times 200$ portion of the spiral in Figure 13: the colors represent the values, ranging from black (smallest) to white (largest).

The method used by James Davis to find $D_{0}$ is quite simple. Suppose $n=m \cdot p$ is fixed by $f$, where $p$ is a prime greater than all the prime factors of $m$. Then $f(n)=f(m) 10^{y}+p$, where $y$ is the number of digits in $p$. From $f(n)=n$ we have $p=\frac{f(m) 10^{y}}{m-1}$. Assuming $p \neq 2,5$, this implies that $p$ divides $f(m)$, and setting $x=f(m) / p$, we find that $m=x 10^{y}+1$ with $p=\frac{f(m)}{x}$ prime. A computer easily finds the solution $x=1407, y=5, m=140700001$, $p=96179$, and so $n=D_{0}$.

No other composite fixed points are known, and David J. Seal has recently shown that there is no composite fixed point less than $D_{0}$. It is easy, however, to find numbers whose trajectory under $f$ ends at $D_{0}$, by repeatedly finding a prime prefix of the previous number, as shown by the example ${ }^{10} D_{1}=13^{532385396179}$ with $f\left(D_{1}\right)=D_{0}$. So presumably there are infinitely many $n$ with $F(n)=-1$.

Consideration of the analogous questions in other bases might have suggested that counterexamples to Conway's question could exist. We will use subscripts to indicate the base

\footnotetext{
${ }^{10}$ Found by Hans Havermann.
} 
(so $\left.4_{10}=100_{2}\right)$. The base-2 analog of $f, f_{2}$ (say), is defined by taking $f_{2}\left(p_{1}^{e_{1}} p_{2}^{e_{2}} \cdots\right)$ to be the concatenation $p_{1} e_{1} p_{2} e_{2} \ldots$, as before (again omitting any $e_{i}$ that are 1 ), except that now we write the $p_{i}$ and $e_{i}$ in base 2 and interpret the concatenation as a base- 2 number. For example, $f_{2}(8)=f_{2}\left(2^{3}\right)=1011_{2}=11_{10}$.

The initial values of $f_{2}(n)(\underline{\mathrm{A} 230625})$ are

$$
\begin{array}{ccccrrrrrrrrrrrrrrrr}
n: & 1 & 2 & 3 & 4 & 5 & 6 & 7 & 8 & 9 & 10 & 11 & 12 & 13 & 14 & 15 & 16 & 17 & 18 & \ldots \\
f_{2}(n): & 1 & 2 & 3 & 10 & 5 & 11 & 7 & 11 & 14 & 21 & 11 & 43 & 13 & 23 & 29 & 20 & 17 & 46 & \ldots
\end{array}
$$

and the base-2 analog of $F, F_{2}(\underline{\mathrm{A} 230627})$ is the prime (or 1) that is reached when $f_{2}$ is repeatedly applied to $n$, or -1 if no prime (or 1 ) is reached:

$$
1,2,3,31,5,11,7,11,23,31,11,43,13,23,29,251,17,23, \ldots \text {. }
$$

Now there is a fairly small composite fixed point, namely 255987, found by David J. Seal. Sean A. Irvine and Chai Wah Wu have also studied this sequence, and the present status is that $F_{2}(n)$ is known for all $n$ less than 12388. All numbers in this range reach 1 , a prime, the composite number 255987 , or one of the two cycles $1007 \longleftrightarrow 1269$ or $1503 \longleftrightarrow 3751$. The numbers for which $F_{2}(n)=-1$ are $217,255,446,558, \ldots$ (A288847). Initially it appeared that 234 might be on this list, but Irvine found that after 104 steps the trajectory reaches the 51-digit prime

350743229748317519260857777660944018966290406786641 .

\section{Home Primes}

A rather older problem arises if we change the definition of $f(n)$ slightly, making $f(8)=222$ rather than 23. So if $n=p_{1} \cdot p_{2} \cdot p_{3} \cdot \ldots$, where $p_{1} \leq p_{2} \leq p_{3} \leq \ldots$, then $f(n)$ is the decimal concatenation $p_{1} p_{2} p_{3} \ldots(\underline{\mathrm{A} 037276})$. In 1990, Jeffrey Heleen studied the analog of $F(n)$ for this function: that is, $F(n)$ is the prime reached if we start with $n$ and repeatedly apply $f$, or -1 if no prime is ever reached (A037274).

The trajectory of 8 now takes 14 steps to reach a prime (the individual prime factors here have been separated by spaces):

$$
\begin{aligned}
8 & \rightarrow 222 \rightarrow 2337 \rightarrow 31941 \rightarrow 33371313 \rightarrow 311123771 \rightarrow 7149317941 \rightarrow \\
& \rightarrow 22931219729 \rightarrow 112084656339 \rightarrow 3347911118189 \rightarrow 11613496501723 \rightarrow \\
& \rightarrow 97130517917327 \rightarrow 531832651281459 \rightarrow 3331113965338635107 \\
& \rightarrow 3331113965338635107,
\end{aligned}
$$

the last number being a prime.

Since $f(n)>n$ if $n$ is composite, now there cannot be any composite fixed points nor any cycles of length greater than 1 . The only way for $F(n)$ to be -1 is for the trajectory of $n$ to be unbounded. This appears to be a harder problem than the one in the previous section, since so far no trajectory has been proved to be unbounded. The first open case is $n=49$, which after 119 iterations has reached a 251-digit composite number (see A056938). The completion of the factorization for step 117 took 765 days by the general number field sieve, and at the time (December 2014) was one of the hardest factorizations ever completed. 


\section{Power Trains}

A third choice for $f(n)$ was proposed by John H. Conway in 2007: he called it the power train map. If the decimal expansion of $n$ is $d_{1} d_{2} d_{3} \ldots d_{k}$ (with $0 \leq d_{i} \leq 9,0<d_{1}$ ), then $f(n)=d_{1}^{d_{2}} \cdot d_{3}^{d_{4}} \cdots$, ending with $\ldots \cdot d_{k}$ if $k$ is odd, or with $\ldots \cdot d_{k-1}^{d_{k}}$ if $k$ is even $(\underline{\mathrm{A} 133500})$.. We take $0^{0}$ to be 1 . For example, $f(39)=3^{9}=19683, f(623)=6^{2} \cdot 3=108$. Conway observed that $2592=2^{5} 9^{2}$ is a non-trivial fixed point, and asked me if there were any others. I found one more: $n=2^{46} \cdot 3^{6} \cdot 5^{10} \cdot 7^{2}=24547284284866560000000000$, for which $f(n)=2^{4} \cdot 5^{4} \cdot 7^{2} \cdot 8^{4} \cdot 2^{8} \cdot 4^{8} \cdot 6^{6} \cdot 5^{6} \cdot 0^{0} \cdot 0^{0} \cdot 0^{0} \cdot 0^{0} \cdot 0^{0}=n$. The eleven known fixed points (including the trivial values $1, \ldots, 9)$ form $\underline{A} 135385$, and it is known that there are no further terms below $10^{100}$. Maybe this is a hint that for all of the functions $f(n)$ that have just been mentioned, there may be only a handful of genuinely exceptional values?

\section{A Memorable Prime}

If you happen to need an explicit 20-digit prime in a hurry, it is useful to remember that although $1,121=11^{2}, 12321=111^{2}, 1234321=1111^{2}, \ldots$, and $12345678987654321=$ $11111111^{2}$ are not primes, the next term in $\underline{\mathrm{A} 173426}$ is a prime,

12345678910987654321 .

As David Broadhurst remarked on the Number Theory Mailing List in August 2015, this is a memorable prime! He also pointed out that on probabilistic grounds, there should be infinitely many values of $n$ such that the decimal concatenation of the numbers 1 up through $n$ followed by $n-1$ down through 1 is a prime. Shortly afterwards, Shyam Sunder Gupta found what is presumably the next prime in the sequence, corresponding to $n=2446$, the 17350-digit probable prime 1234567..244524462445..7654321. Serge Batalov has shown that there are no further terms with $n<60000$. What is the next term? The values 10, 2446 are not enough to create an OEIS entry.

\section{A Missing Prime}

The previous question naturally led me to wonder what the first prime is in the simpler sequence $(\underline{\mathrm{A} 007908)})$ :

$$
1,12,123,1234, \ldots, 12345678910,1234567891011, \ldots,
$$

formed by the decimal concatenation of the numbers 1 through $n$. In Unsolved Problems in Number Theory, Richard K. Guy reports that this question was already asked by Charles Nicol and John Selfridge. However, although the same probabilistic argument suggests that there should be an infinite number of primes of this type, not a single one is known. I asked several friends to help with the search, and as a result this task was taken up by the folks who run the GIMP (or Great Internet Mersenne Prime) search, and there is now a web page ${ }^{11}$

\footnotetext{
${ }^{11}$ http://mersenneforum. org/showthread.php?t=20527.
} 
that shows the current status of the search for the first prime. As of August 2017 the search seems to have stalled, the present status being that all the potential values of $n$ through 344869 failed (obviously many values of $n$ can be ruled out by congruence conditions). In this range the candidates have about two million digits. One estimate suggests that there is a probability of about 0.5 that a prime will be found with $n<10^{6}$, so it would be good to resume this search.

\section{Post's Tag System}

In his recent book Elements of Mathematics: From Euclid to Gödel ${ }^{12}$, John Stillwell mentions that Emil L. Post's tag system from the 1930s is still not understood. Post asked the following question. Take a finite string, or word, $S$ of 0 s and $1 \mathrm{~s}$, and if it begins with 0 , append 00 to the end of $S$ and delete the first three symbols, or if it begins with 1, append 1101 to the end of $S$ and delete the first three symbols. When this process is iterated, eventually one of three things will happen: either $S$ will reach the empty word ( $S$ dies), $S$ will enter a loop ( $S$ cycles), or $S$ will keep growing for ever $(S$ blows up). For example, $S=1000$ reaches the empty word $\epsilon$ at the 7 th step:

$$
1000 \rightarrow 01101 \rightarrow 0100 \rightarrow 000 \rightarrow 00 \rightarrow 0 \rightarrow \epsilon
$$

whereas 100100 enters a cycle of length six (indicated by parentheses) after 15 steps:

$$
\begin{aligned}
100100 & \rightarrow 1001101 \rightarrow 11011101 \rightarrow 111011101 \rightarrow 0111011101 \rightarrow 101110100 \rightarrow 1101001101 \\
& \rightarrow 10011011101 \rightarrow 110111011101 \rightarrow 1110111011101 \rightarrow 01110111011101 \\
& \rightarrow 1011101110100 \rightarrow 11011101001101 \rightarrow 111010011011101 \rightarrow 0100110111011101 \\
& \rightarrow(011011101110100 \rightarrow 01110111010000 \rightarrow 1011101000000 \rightarrow 11010000001101 \\
& \rightarrow 100000011011101 \rightarrow 0000110111011101)
\end{aligned}
$$

Post was hoping to find an algorithm which, given $S$, would determine which of these outcomes would occur. He did not succeed.

Post called this process a 'tag system.' It can be generalized by considering initial words over an alphabet of size $M$ (rather than 2), allowing any fixed set $\mathcal{A}$ of $M$ tag words to be appended (rather than 00 and 1101), and deleting some fixed number $P$ of initial symbols at each step (not necessarily 3). In 1961, Marvin Minsky showed that such a generalized tag system could simulate a Turing machine. By choosing an appropriate alphabet, an appropriate set $\mathcal{A}$ of tag words to be appended, and an appropriate value of $P$ (in fact $P=2$ will do), any computable function can be simulated. So, because of the undecidability of the Halting Problem, for general tag systems it is impossible to predict which initial words will blow up.

But what about Post's original tag system? Could this simulate a Turing machine (by encoding the problem in the initial word $S)$ ? At first this seems very unlikely, but the Cook-Wolfram theorem that the one-dimensional cellular automaton defined by Rule 110

\footnotetext{
${ }^{12}$ A superb successor to Felix Klein's 1908 Elementary Mathematics from an Advanced Standpoint.
} 
can simulate a Turing machine (by encoding the problem in the starting state) suggests that it might be possible. If it is possible, there must be some initial words that blow up (again because of the Halting Problem).

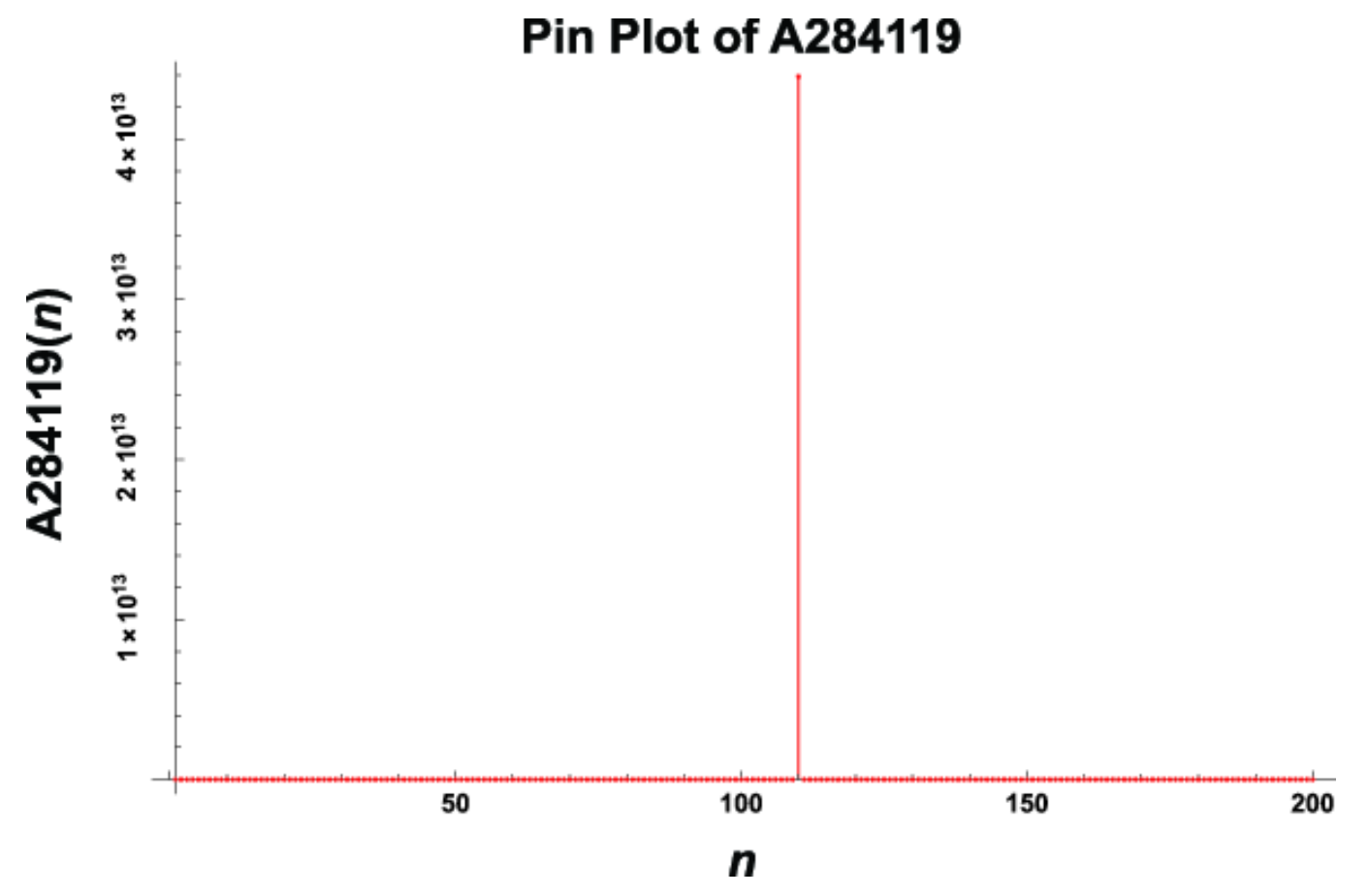

Figure 15: Pin plot illustrating Lars Blomberg's remarkable discovery that the Post tag system started at the word $(100)^{110}$ takes an exceptionally long time (43913328040672 steps) to converge.

In early 2017, when I read Stillwell's book,, the OEIS contained three sequences related to the original tag system, based on the work of Peter Asveld and submitted by Jeffrey Shallit: A284116, giving the maximal number of words in the 'trajectory' of any initial word $S$ of length $n$ (18 terms were known), and two sequences connected with the especially interesting starting word $\sigma_{n}$ of length $3 n$ consisting of $n$ copies of 100 . A284119 $(n)$ is defined to be the number of words in the trajectory of $\sigma_{n}$ before it enters a cycle or dies, or -1 if the trajectory blows up, and A284121 $(n)$ is the length of the cycle, or 1 if the trajectory dies, or -1 if the trajectory blows up. For example, from $(5)$ we see that $\underline{A} 284119(2)=15$ and A284121 $(2)=6$. Shallit had extended Asveld's work and had found 43 terms of the two last-mentioned sequences.

I then added many further sequences based on tag systems discussed by Asveld, Liesbeth De Mol, Shigeru Watanabe, and others, and appealed to contributors to the OEIS to extend them.

The most interesting response came from Lars Blomberg, who investigated the trajectory of $\sigma_{n}$ for $n \leq 110$. On September 92017 he reported that every $\sigma_{n}$ for $n \leq 110$ had either died or cycled after at most 13 million terms, except for $\sigma_{110}$, which after $38.10^{11}$ steps had reached a word of length $10^{7}$ and was still growing. This was exciting news! Could $\sigma_{110}$ be 
the first word to be discovered that blew up? ${ }^{13}$ Sadly, on October 4 2017, Blomberg reported that after 43913328040672 steps $\sigma_{110}$ had terminated in the empty word.

Figure 15 displays the remarkable graph (technically, a pin plot) of the number of steps for $\sigma_{n}$ to either die or cycle for $n \leq 200$. Figure 16 shows the lengths of the successive words in the trajectory of $\sigma_{110}$.

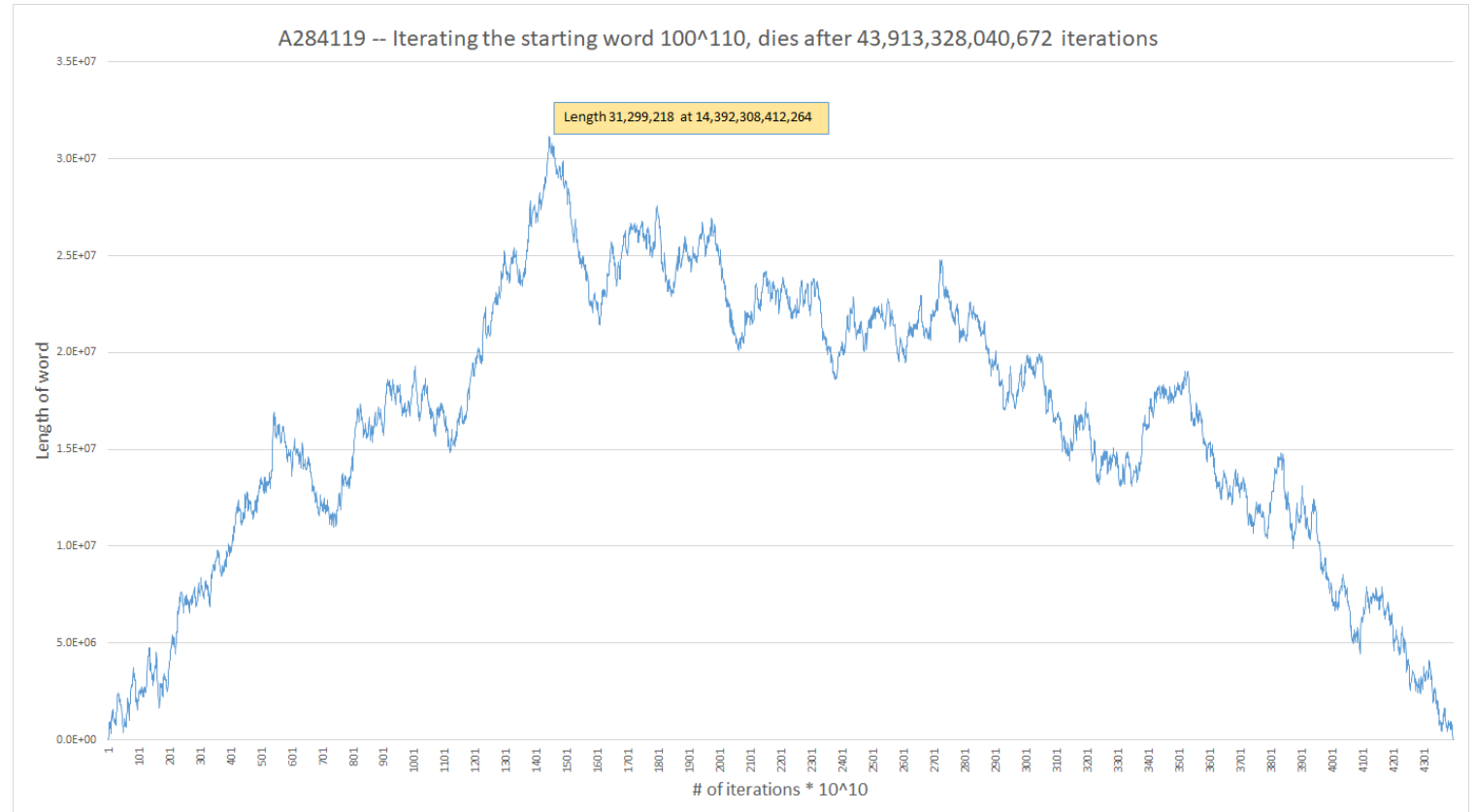

Figure 16: Lengths of successive words in trajectory of $(100)^{110}$ under the Post tag system. The numbers on the horizontal axis are spaced at multiples of $10^{12}$.

In the past six months Blomberg has continued this investigation and has determined the fate of $\sigma_{n}$ for all $n \leq 6075$. The new record-holder for the number of steps before the trajectory dies is now held by $\sigma_{4974}$, which takes 57042251906801 steps, while $\sigma_{110}$ is in second place.

Of course it is still possible that some initial word $S$, not necessarily of the form $\sigma_{n}$, will blow up, but this seems increasingly unlikely. So Post's tag system probably does not simulate a Turing machine.

The question as to which $\sigma_{n}$ die and which cycle remains a mystery. Up to $n=6075$, Blomberg's results show that about one-sixth of the values of $n$ die and five-sixths cycle. The precise values can be found in A291792. It would be nice to understand this sequence better.

\footnotetext{
${ }^{13}$ Of course the fact that the same number 110 was involved could not possibly be anything more than a coincidence.
} 


\section{Coordination Sequences}

This final section is concerned with coordination sequences, which arise in crystallography and in studying tiling problems, have beautiful illustrations, and lead to many unsolved mathematical questions.

The "Cairo" tiling, so called because it is said to be used on many streets in that city, is shown in Figure 17. Let $G$ denote the corresponding infinite graph (with vertices for points where three or more tiles meet, and edges between two vertices where two tiles meet). The figure is also a picture of the graph.

The distance between vertices $P, Q \in G$ is defined to be the number of edges in the shortest path joining them. The coordination sequence of $G$ with respect to a vertex $P \in G$ is then the sequence $a(n)(n \geq 0)$ giving the number of vertices $Q$ at distance $n$ from $P$. Coordination sequences have been studied by crystallographers for many years [5].

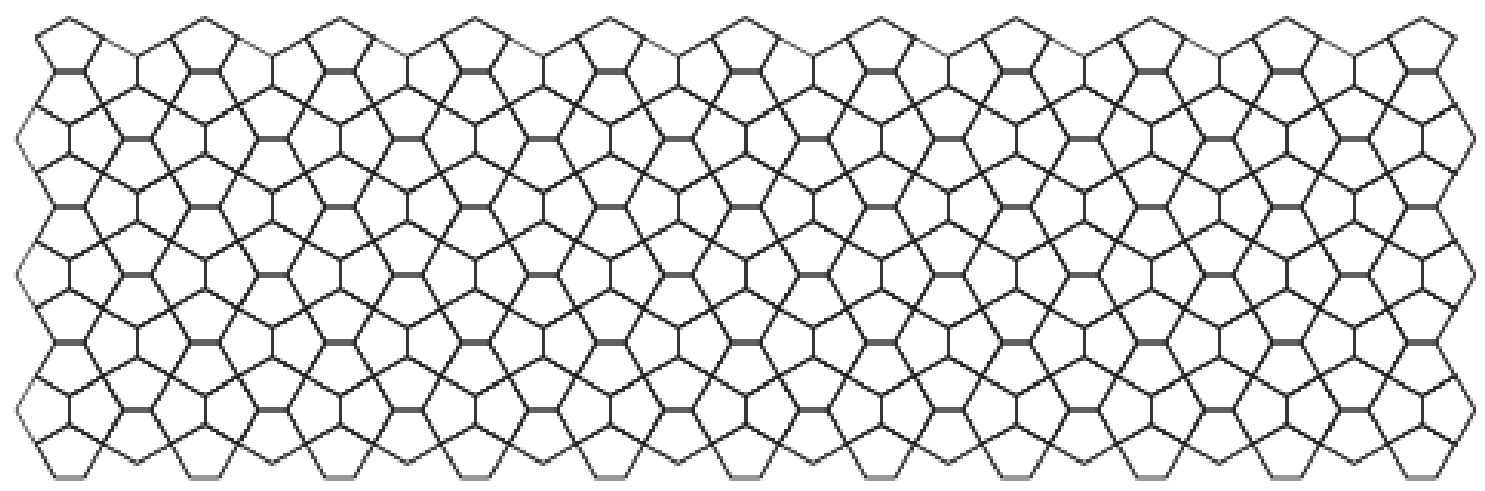

Figure 17: A portion of the Cairo tiling.

The graph of the Cairo tiling has two kinds of vertices, trivalent (where three edges meet) and tetravalent. As can be seen from Figure 17, the coordination sequence with respect to a tetravalent vertex begins $1,4,8,12,16,20,24, \ldots$, which appears to be the same as the coordination sequence A008574 for a vertex in the familiar square grid. This observation seemed to be new. Chaim Goodman-Strauss and I thought that such a simple fact should have a simple proof, and we developed an elementary "coloring book" procedure [4] which not only proved this result but also established a number of conjectured formulas for coordination sequences of other tilings mentioned in entries in the OEIS. The "coloring book" drawing of the Cairo graph of the Cairo graph centered at a tetravalent vertex is shown in Figure 18. This coloring makes it easy to prove that the coordination sequence is given by $a(n)=4 n$ for $n \geq 1$ (see [4] for details).

For a trivalent vertex in the Cairo tiling, the coordination sequence is

$$
1,3,8,12,15,20,25,28,31,36,41,44,47,52,57,60,63,68, \ldots
$$

(this is now A296368), and we [4] show that for $n \geq 3, a(n)=4 n$ if $n$ is odd, $4 n-1$ if $n \equiv 0$ $(\bmod 4)$, and $4 n+1$ if $n \equiv 2(\bmod 4)$.

One can similarly define coordination sequences for other two- and higher-dimensional structures, and the OEIS presently contains over 1300 such sequences. Many more could be 


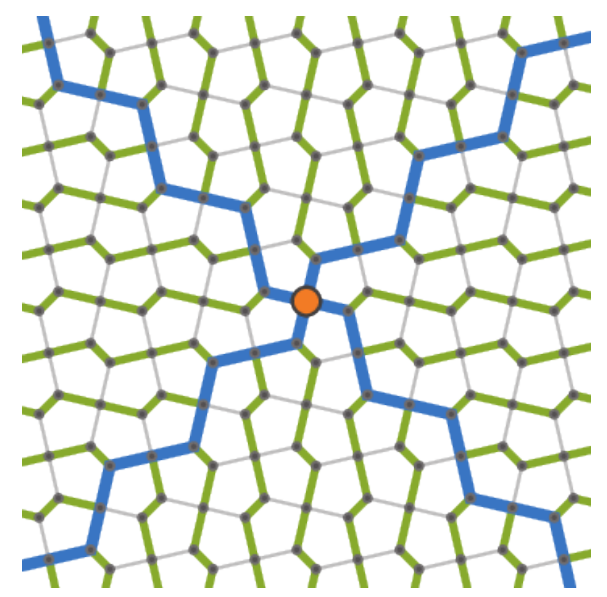

Figure 18: The "coloring book" method applied to a tetravalent vertex (the red dot) in the Cairo tiling, used to prove that the coordination sequence is the same as that for the square grid.

added. There are many excellent web sites with lists of tilings and crystals. Brian Galebach's web site ${ }^{14}$ is especially important, as it includes pictures of all " $k$-uniform" tilings with $k \leq 6$, with over 1000 tilings. Darrah Chavey's article [2] and the Michael Hartley and Printable Paper web sites ${ }^{15}$ have many further pictures, and the RCSR and ToposPro databases ${ }^{16}$ have thousands more.

Only last week (on May 4, 2018), Rémy Sigrist investigated the Ammann-Beenker (or "octagonal") tiling shown in Figure 19, an aperiodic tiling with eight-fold rotational symmetry about the central point.

Sigrist determined the initial terms of the coordination sequence with respect to the central vertex (A303981):

$$
1,8,16,32,32,40,48,72,64,96,80,104,112,112,128,152, \ldots
$$

Figure 20 shows the vertices at distances $0,1,2, \ldots, 6$ from the center.

No formula or growth estimate is presently known for this sequence. However, earlier this year Anton Shutov and Andrey Maleev determined the asymptotic behavior of the coordination sequence $(\underline{\text { A302176 }})$ with respect to a vertex with five-fold rotational symmetry in a certain Penrose tiling. So we end with a question: Can the Shutov-Maleev approach be used to find the asymptotic growth of (6)? Of course an explicit formula would be even nicer.

\footnotetext{
${ }^{14}$ http://probabilitysports.com/tilings.html.

15 http://www.dr-mikes-math-games-for-kids.com/archimedean-graph-paper.html, https://www . printablepaper . net/category/graph.

${ }^{16}$ http://rcsr.net, http://topospro.com.
} 


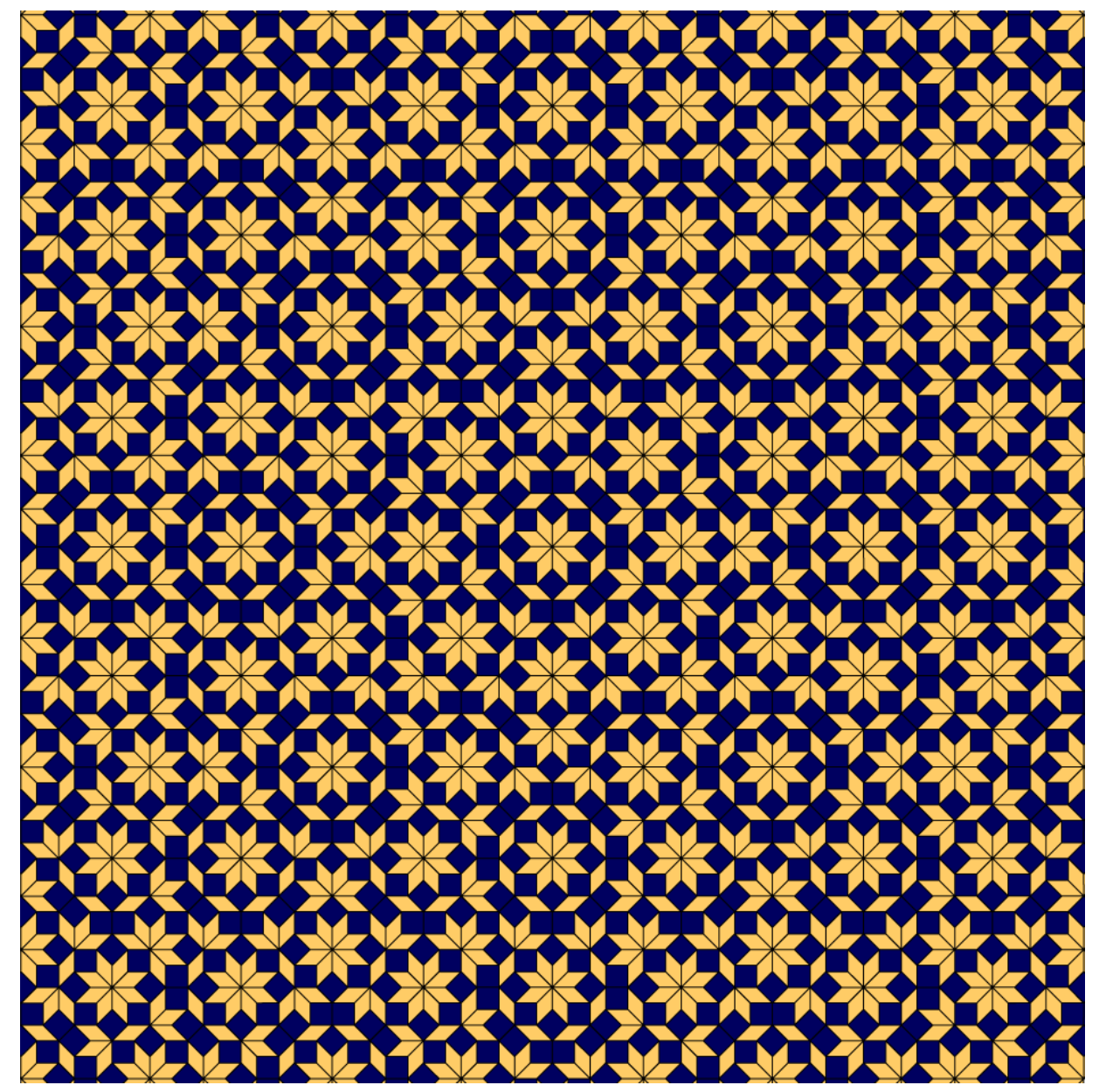

Figure 19: The Ammann-Beenker or "octahedral" tiling.

\section{Acknowledgments}

Now that the OEIS is a wiki, many volunteer editors help maintain it. ${ }^{17}$ Besides the OEIS contributors already mentioned in this article, I would like to thank Jörg Arndt, Michael De Vlieger, Charles Greathouse IV, Alois P. Heinz, Michel Marcus, Richard J. Mathar, Don Reble, Jon E. Schoenfield, and Allan C. Wechsler for their help. (Many other names could be added to this list.)

\section{References}

[1] D. L. Applegate, H. Havermann, B. Selcoe, V. Shevelev, N. J. A. Sloane, and R. Zumkeller, The Yellowstone Permutation, J. Integer Seqs., 18 (2015), \#15.6.7.

[2] D. Chavey, Tilings by regular polygons II: A catalog of tilings, Computers \& Mathematics with Applications, 17:1-3 (1989), 147-165.

\footnotetext{
${ }^{17}$ And more are needed - if interested, please contact the author.
} 


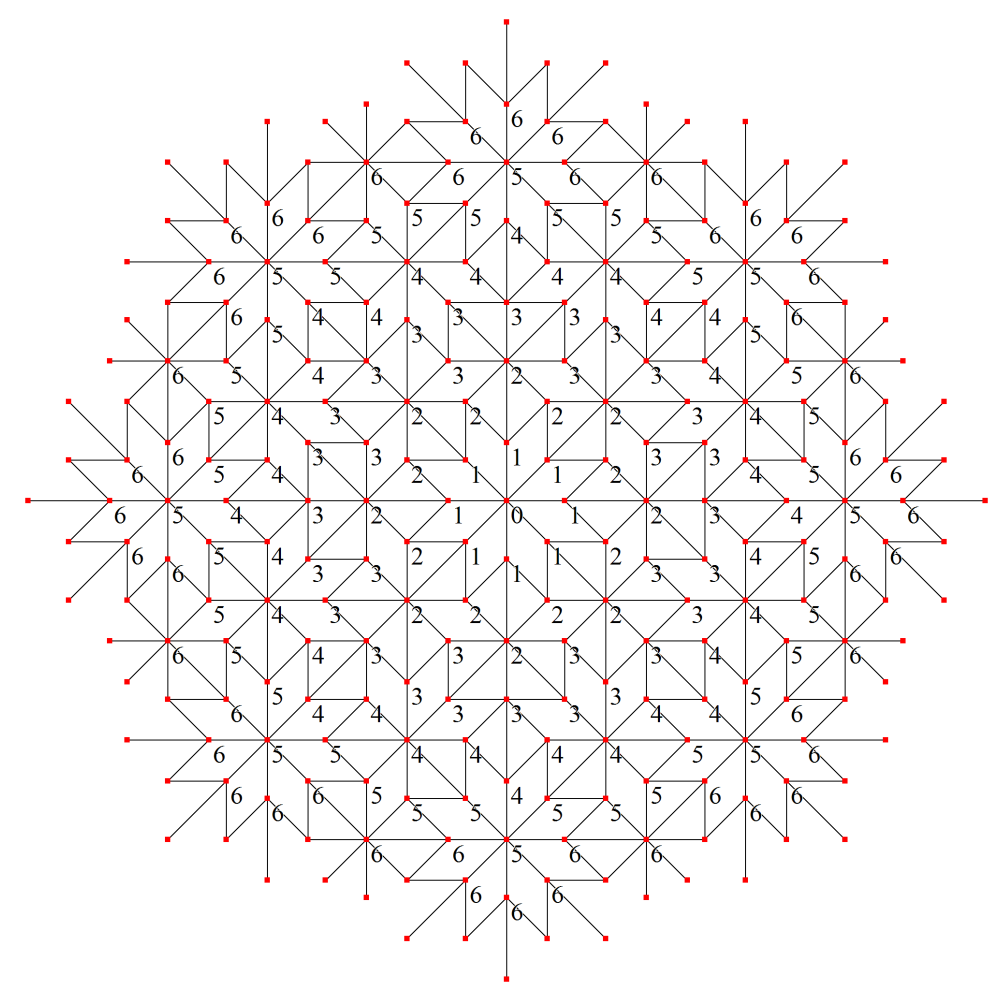

Figure 20: Illustrating the coordination sequence for the Ammann-Beenker tiling, showing the vertices at distances 0 though 6 from the central vertex.

[3] P. Erdős, R. Freud, and N. Hegyvári, Arithmetical properties of permutations of integers, Acta Math. Hungar., 41 (1983), 169-176.

[4] C. Goodman-Strauss and N. J. A. Sloane, A coloring book approach to finding coordination sequences, arXiv preprint, arXiv:1803.08530, March 2018.

[5] M. O'Keeffe and B. G. Hyde, Plane nets in crystal chemistry, Phil. Trans. Royal Soc. London, Series A, Mathematical and Physical Sciences, 295:1417 (1980), 553-618.

[6] P. Vanderlind, R. K. Guy, and L. C. Larson, The Inquisitive Problem Solver, MAA Press, 2002.

\section{ABOUT THE AUTHOR}

Neil J. A. Sloane started collecting sequences in 1964. Every advance in computing since then has made the task easier, and a subtitle could be "From punched cards to wiki in 54 years." The Unix operating system was the greatest breakthrough of all, especially grep, the programmable editor $e x$, and pipes.

Image Credits 
- Figures 1-5, 10, 11, 13, 15: Michael De Vlieger (who also designed the "Peace to the Max" T-shirt in Figure 3).

- Figure 6: Jessica Gonzalez.

- Figures 7-9: Jonathan Wild.

- Figure 12: Hans Havermann.

- Figure 14: Kerry Mitchell.

- Figure 16: Lars Blomberg.

- Figures 17, 18: Chaim Goodman-Strauss.

- Figure 19: The Tilings Encyclopedia.

- Figure 20: Rémy Sigrist. 